\title{
CLINICAL ANALYSIS OF SMALL-SIZED PERIPHERAL LUNG CANCER
}

Teruaki Koike, $\mathrm{MD}^{\mathrm{a}}$

Masanori Terashima, MD $^{\mathrm{a}}$

Tsuneyo Takizawa, MD ${ }^{\mathrm{a}}$

Takehiro Watanabe, $\mathrm{MD}^{\mathrm{a}}$

Yuzo Kurita, $\mathrm{MD}^{\mathrm{b}}$

Akira Yokoyama, $\mathrm{MD}^{\mathrm{b}}$
Objective: In Japan, with the initiation of the lung cancer screening program, small-sized peripheral lung cancer in which the diameter is $2 \mathrm{~cm}$ or less has been increasing. The purpose of this study is to determine the clinicopathologic behavior of small-sized lung cancer. Methods: Four hundred ninety-six patients with cT1 N0, peripheral, resected non-small-cell lung cancer, who were operated on between 1980 and 1996, were selected, grouped by tumor diameter or histologic type, and then analyzed for clinicopathologic behavior. On the basis of measured diameter roentgenographically, the patients were divided into two groups; group c-S with lesions $2 \mathrm{~cm}$ or less in diameter and group c-L with lesions 2.1 to $3 \mathrm{~cm}$ in diameter. Results: Lymph node metastasis was recognized in $18 \%$ of group $\mathrm{c}-\mathrm{S}$, in $\mathbf{2 3} \%$ of group c-L, and in $\mathbf{2 1 \%}$ for the entire clinical group. The rate of those with the progressive state was $19 \%$ in group c-S and $26 \%$ in group c-L. The 5-year survival was $79.5 \%$ in group c-S and $69.3 \%$ in group c-L (i.e., there was a significant difference between the two groups). Conclusion: Compared with the patients with lesions 2.1 to $3 \mathrm{~cm}$ in diameter, the patients with small-sized lung cancer had a milder progressive state and a better prognosis. (J Thorac Cardiovasc Surg 1998;115:1015-20)
$\mathrm{R}$ ecently in Japan, with the spread of mass screening for lung cancer, there have been many opportunities to resect small-sized peripheral lung cancers with diameters of $2 \mathrm{~cm}$ or less. Occasionally it is difficult to decide whether we should perform lobectomy or add systematic lymphadenectomy in patients with small-sized lung cancers. As these small-sized lung cancers increase, however, it becomes important to consider the clinicopathologic behavior of these tumors and to determine an optimal treatment strategy. In our institute, patients with lung cancer have usually been treated with lobectomy and added systematic lymphadenectomy, and recently we began to apply limited resection to a few patients with small-sized lung cancer. Thus we

From the Division of Chest Surgery a and the Division of Medi-

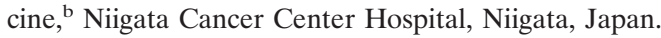

Received for publication July 16, 1997; revisions requested Oct. 14, 1997; revisions received Dec. 15, 1997; accepted for publication Dec. 15, 1997.

Address for reprints: Teruaki Koike, MD, Division of Chest Surgery, Niigata Cancer Center Hospital, 2-15-3, Kawagishicho, Niigata 951, Japan.

Consultant statistician: Koichi Inada, DSc, Department of Mathematics and Computer Science, Faculty of Science, Kagoshima University, Kagoshima 890, Japan.

Copyright (C) 1998 by Mosby, Inc.

0022-5223/98 $\$ 5.00+0 \quad \mathbf{1 2 / 1 / 8 8 2 1 0}$ can reliably detect the intrathoracic pathologic spread of these small-sized lung cancers. The purpose of this study is to determine the clinicopathologic behavior of small-sized lung cancer retrospectively.

\section{Patients and methods}

After the introduction of chest computed tomographic scanning for preoperative clinical staging, patients with resected primary lung cancer treated at our institution from January 1980 to December 1996 were the subjects of this study. The following criteria were used. Peripheral cT1 N0 M0 lung cancer with a maximum diameter of $3 \mathrm{~cm}$ or less, measured on plain chest x-ray films or computed tomographic scans, were the first criterion. The staging of lung cancer was based on the TNM classification of the Union International Cancer Conference. ${ }^{1}$ The second criterion was that the patients had no former treatment. The third criterion was that the histologic type be nonsmall-cell lung cancer, except small-cell lung cancer or low-grade malignant tumors. The fourth criterion was that lobectomy had been performed with complete hilar and mediastinal lymphadenectomy. A total of 496 patients were selected, 255 of whom were men and 241 were women. They ranged in age from 29 to 83 years (mean age 63 years). The selected patients were separated clinically into two subgroups by tumor diameter; the small group (group c-S) consisted of patients in whom the tumor diameter measured on plain chest x-ray films or by computed tomographic scanning was $2 \mathrm{~cm}$ or less, and the large group (group c-L) consisted of patients in which the diameter was 2.1 to $3 \mathrm{~cm}$. The patients were classified into 


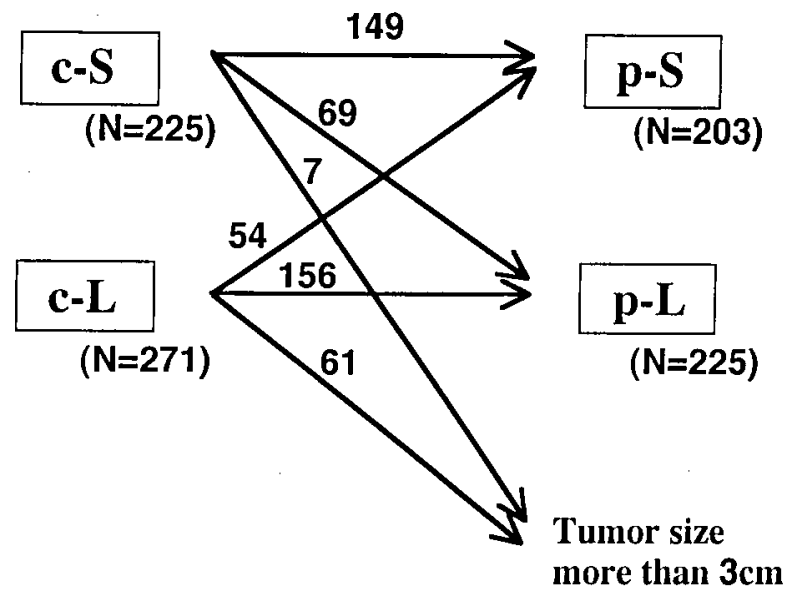

Fig. 1. Schema of clinical and postoperative groups. Group c-S consisted of cases with a maximum tumor diameter as measured by roentgenography or computed tomography of $2 \mathrm{~cm}$ or less. Group c-L consisted of cases with a roentgenographic tumor diameter of 2.1 to $3 \mathrm{~cm}$. Group p-S consisted of cases in which the resected specimen diameters were $2 \mathrm{~cm}$ or less, and group $\mathrm{p}-\mathrm{L}$ consisted of cases in which the resected specimen diameters were 2.1 to $3 \mathrm{~cm}$.

another two subgroups based on the resected tumor diameter; the postoperative small group (group p-S) consisted of patients with tumors $2 \mathrm{~cm}$ or less in diameter, and the postoperative large group (group p-L) consisted of patients with tumors 2.1 to $3 \mathrm{~cm}$ in diameter (Fig. 1). Among 225 group c-S patients, 149 (66\%) in whom the resected tumor diameter was $2 \mathrm{~cm}$ or less were classified as group p-S, and in 271 group c-L patients, $156(58 \%)$ in whom the resected tumor diameter was 2.1 to $3 \mathrm{~cm}$ were classified into group $\mathrm{p}-\mathrm{L}$. Three hundred five patients (61\%) showed a match between clinical diameter and postopertive diameter, 54 patients $(11 \%)$ overestimation, and 137 patients $(28 \%)$ underestimation.

Statistical analysis was carried out using the $\chi^{2}$ test. Survival was calculated by the Kaplan-Meier life-table method and compared with the generalized Wilcoxon test. Multivariance analysis of the prognostic factors was conducted by Cox's regression model. It was performed with SAS software (SAS Institute, Inc. Cary, N.C.).

\section{Results}

When the tumor cell cytologic findings were judged to be class IV or V, lung cancer was diagnosed. In $90 \%$ of the patients, lung cancer was diagnosed before the operation (Table I). The positive rate was $92 \%$ in group c-L and $87 \%$ in group $\mathrm{c}-\mathrm{S}$, showing a decrease with the reduction in tumor diameter. At our institution, to obtain tumor cells, we first examined patients with a flexible fiberoptic bronchoscope. If no positive tumor cells were ob-
Table I. Preoperative diagnosis: clinical group

\begin{tabular}{cccc}
\hline Group & $c-S$ & $c-L$ & $c$-Group \\
$(N=225)$ & $(N=271)$ & $(N=496)$ \\
\hline Diagnosed cases & $195(87 \%)$ & $250(92 \%)$ & $445(90 \%)$ \\
FB & $168(75 \%)$ & $232(85 \%)$ & $400(81 \%)$ \\
NB & $27(12 \%)$ & $18(7 \%)$ & $45(9 \%)$
\end{tabular}

$c-S$, Clinical small (roentgenographically measured tumor diameter $2 \mathrm{~cm}$ or less); $c-L$, clinical large (roentgenographically measured tumor diameter 2.1 to $3 \mathrm{~cm}$ ); $c$-Group, c-S + c-L; FB, fiberoptic bronchoscopy; $N B$, needle biopsy.

Table II. Pathologic stage according to tumor diameter

\begin{tabular}{lcccccc}
\hline \multicolumn{7}{c}{ Group } \\
\cline { 2 - 7 } & $c-S$ & $c-L$ & $c$-Group & $p-S$ & $p-L$ & $p$-Group \\
Stage & $(\%)$ & $(\%)$ & $(\%)$ & $(\%)$ & $(\%)$ & $(\%)$ \\
\hline I & $184(82)$ & $201(74)$ & $385(78)$ & $164(83)$ & $171(76)$ & $339(79)$ \\
II & $16(7)$ & $21(8)$ & $37(7)$ & $12(6)$ & $21(9)$ & $33(8)$ \\
IIIA & $21(10)$ & $38(14)$ & $59(12)$ & $18(9)$ & $24(13)$ & $47(11)$ \\
IIIB & $3(1)$ & $10(4)$ & $13(3)$ & $3(1)$ & $4(2)$ & $7(2)$ \\
IV & $1(0)$ & $1(0)$ & $2(0)$ & $2(1)$ & $0(0)$ & $2(0)$ \\
\hline
\end{tabular}

$c-S$, Clinical small (roentgenographically measured tumor diameter $2 \mathrm{~cm}$ or less); $c-L$, clinical large (roentgenographically measured tumor diameter 2.1 to $3 \mathrm{~cm}$ ); $c$-Group, c-S + c-L; $p$-S, postoperative small (tumor diameter of resected specimen $2 \mathrm{~cm}$ or less); $p-L$, postoperative large (tumor diameter of resected specimen 2.1 to $3 \mathrm{~cm}$ ); p-Group, $\mathrm{p}-\mathrm{S}+\mathrm{p}$-L.

tained, we attempted percutaneous needle biopsy. Eighty-one percent of cases were diagnosed by examination with fiberoptic bronchoscopy and $9 \%$ by needle biopsy. The positive rate by needle biopsy in group c-S was higher than that in group c-L. Thus for small-sized lung cancer the needle biopsy examination appears to increase the positive rate.

According to the classification by pathologic staging, p stage I was $82 \%$ in group c-S and $74 \%$ in group c-L (Table II). The rate of p stage I in group c-S was higher than in group c-L. This tendency was also seen in the postoperative group; p stage I was $83 \%$ in group $\mathrm{p}-\mathrm{S}$ and $76 \%$ in group $\mathrm{p}-\mathrm{L}$.

Lymph node metastasis was recognized histologically in $18 \%$ of group c-S, $23 \%$ of group c-L, and $21 \%$ of the entire clinical group, whereas intrapulmonary metastasis was recognized in $1 \%$ of group c-S, $2 \%$ of group c-L, and $2 \%$ of the clinical group (Table III). Pleural dissemination or malignant effusion was recognized histologically in $1 \%$ of group c-S, $3 \%$ of group $\mathrm{c}-\mathrm{L}$, and $2 \%$ of the clinical group. If there are certain progressive conditions, such as lymph node metastasis, intrapulmonary metastasis, pleural dissemination, or malignant effusion, limited resection increases the risk of incomplete resection. Among 225 group c-S patients, lymph node metastasis alone was recognized in 
Table III. Pathologic confirmation according to tumor diameter

\begin{tabular}{lcccccc}
\hline & \multicolumn{9}{c}{ Group } \\
\cline { 2 - 7 } & $c-S$ & $c-L$ & $c$-Group & $p$-S & $p$ - $L$ & $\begin{array}{c}p \text {-Group } \\
(N=428)\end{array}$ \\
& $(N=225)$ & $(N=271)$ & $(N=496)$ & $(N=203)$ & $(N=225)$ & $85(20 \%)$ \\
$\mathrm{n}(+)$ & $40(18 \%)$ & $63(23 \%)$ & $103(21 \%)$ & $34(17 \%)$ & $51(23 \%)$ & $7(2 \%)$ \\
$\mathrm{pm}(+)$ & $2(1 \%)$ & $6(2 \%)$ & $8(2 \%)$ & $3(1 \%)$ & $4(2 \%)$ & $7(2 \%)$ \\
$\mathrm{D} / \mathrm{E}(+)$ & $3(1 \%)$ & $9(3 \%)$ & $12(2 \%)$ & $3(1 \%)$ & $4(2 \%)$ & $92(21 \%)$ \\
$\mathrm{Incomp}$ & $43(19 \%)$ & $70(26 \%)$ & $113(23 \%)$ & $37(18 \%)$ & $55(24 \%)$ &
\end{tabular}

$c-S$, Clinical small (roentgenographically measured tumor diameter $2 \mathrm{~cm}$ or less); $c-L$, clinical large (roentgenographically measured tumor diameter 2.1 to $3 \mathrm{~cm}$ ); $c$-Group, c-S + c-L; $p$-S, postoperative small (tumor size of resected specimen $2 \mathrm{~cm}$ or less); $p$ - $L$, postoperative large (tumor size of resected specimens 2.1 to $3 \mathrm{~cm}$ ); $p$-Group, p-S + p-L; $n$, lymph node metastasis; $p m$, intrapulmonary metastasis; $D / E$, dissemination or malignant effusion; Incomp, incomplete risk rate after limited resection for cases with lymph node metastasis, pulmonary metastasis, pleural dissemination, or malignant effusion.

Table IV. Pathologic confirmation according to histologic type

\begin{tabular}{llclll}
\hline & \multicolumn{2}{c}{ Clinical group } & & \multicolumn{2}{c}{ Postoperative group } \\
\cline { 2 - 3 } & Sq. ca. & Ad. ca. & Others & Sq. ca. & Ad. ca. \\
& $(N=64)$ & $(N=422)$ & $(N=10)$ & $(N=49)$ & $(N=369)$ \\
\hline $\mathrm{n}(+)$ & $7(11 \%)$ & $92(22 \%)$ & $4(40 \%)$ & $5(10 \%)$ & $76(21 \%)$ \\
$\mathrm{pm}(+)$ & $0(0 \%)$ & $8(2 \%)$ & $0(0 \%)$ & $0(0 \%)$ & $7(2 \%)$ \\
$\mathrm{D} / \mathrm{E}(+)$ & $0(0 \%)$ & $12(3 \%)$ & $0(0 \%)$ & $0(0 \%)$ & $7(2 \%)$ \\
Incomp. & $7(11 \%)$ & $102(24 \%)$ & $4(40 \%)$ & $5(10 \%)$ & $83(22 \%)$ \\
\hline
\end{tabular}

Sq. ca., Squamous cell carcinoma; $A d$. ca., adenocarcinoma; Others, large cell carcinoma + adenosquamous cell carcinoma; $n$, lymph node metastasis; $p m$, intrapulmonary metastasis; $D / E$, dissemination or malignant effusion; Incomp., incomplete risk rate after limited resection for cases with lymph node metastasis, pulmonary metastasis, pleural dissemination, or malignant effusion.

38 patients, intrapulmonary metastasis alone in 1 patient, and malignant effusion alone in 2 patients. Lymph node metastasis and intrapulmonary metastasis were recognized concurrently in one patient, and lymph node metastasis and malignant effusion were found concurrently in one patient. Therefore the risk of incomplete resection was suspected to be approximately $19 \%$ in group c-S and $26 \%$ in group c-L. The same tendency was seen in postoperative tumor diameter. The incidence rates of lymph node metastasis, intrapulmonary metastasis, pleural dissemination, and malignant effusion in group $\mathrm{p}-\mathrm{L}$ were higher than those in group $\mathrm{p}-\mathrm{S}$. Thus the risk of incomplete resection in $\mathrm{p}-\mathrm{L}$ was higher than that in $\mathrm{p}-\mathrm{S}$.

In the clinical group, according to the classification of histologic types, lymph node metastasis was recognized in $11 \%$ of squamous cell carcinoma, $22 \%$ of adenocarcinoma, and $40 \%$ of other cell types (five patients had large cell carcinoma and five patients had adenosquamous cell carcinoma) (Table IV). Intrapulmonary metastasis was recognized in only $2 \%$ of patients with adenocarcinoma, pleural dissemination or malignant effusion in only $3 \%$ of these patients. The risk of incomplete resection was $11 \%$
Table V. Lymph node metastasis according to tumor diameter and histologic type

\begin{tabular}{lccr}
\hline \multicolumn{4}{c}{ Clinical group } \\
\hline & $c-S$ & $c-L$ & $c$-Group \\
& $(\%)$ & $(\%)$ & $(\%)$ \\
\hline Sq. ca. & $2 / 28(7)$ & $5 / 36(14)$ & $7 / 64(11)$ \\
Ad. ca. & $36 / 193(19)$ & $56 / 229(24)$ & $92 / 422(22)$ \\
\hline \multicolumn{4}{c}{ Postoperative group } \\
\hline \multicolumn{4}{c}{$p-L$} \\
\hline Sq. ca. & $p-S$ & $(\%)$ & $p$-Group \\
Ad. ca. & $29 / 27(171(17)$ & $1 \%)$ & $5 / 49(10)$ \\
\hline
\end{tabular}

$c-S$, Clinical small (roentgenographically measured tumor diameter $2 \mathrm{~cm}$ or less); $c-L$, clinical large (roentgenographically measured tumor diameter 2.1 to $3 \mathrm{~cm}$ ); $c$-Group, c-S + c-L; $p$-S, postoperative small (tumor diameter of resected specimen $2 \mathrm{~cm}$ or less); $p-L$, postoperative large (tumor diameter of 2.1 to $3 \mathrm{~cm}$ ); p-Group, p-S + p-L; Sq. ca., squamous cell carcinoma; $A d$. ca., adenocarcinoma.

in squamous cell carcinoma, $24 \%$ in adenocarcinoma, with the risk rate in adenocarcinoma significantly higher than that in squamous cell carcinoma $(p=0.02)$.

In the other subgroups of the clinical group, rates were the same as in the postoperative group. In the postoperative group, lymph node metastasis was recognized in $10 \%$ of squamous cell carcinoma, $24 \%$ of adenocarcinoma, and $2 \%$ each of intrapulmonary metastasis and pleural dissemination or malignant effusion with adenocarcinoma. The risk of incomplete resection was $10 \%$ for squamous cell carcinoma and $22 \%$ for adenocarcinoma.

In the subgroups classified by histologic type and tumor size, the rate of lymph node metastasis of adenocarcinoma was higher than that of squamous cell carcinoma in both the clinical and the postoperative group (Table V). For adenocarcinoma, the positive rate of group c-S was lower than that of group c-L, and the positive rate of group $\mathrm{p}-\mathrm{S}$ was 
Table VI. Risk of incomplete resection according to tumor diameter and histologic type

\begin{tabular}{lcccccc}
\hline Group & $\begin{array}{c}c-S \\
(\%)\end{array}$ & $\begin{array}{c}c-L \\
(\%)\end{array}$ & $\begin{array}{c}c \text {-Group } \\
(\%)\end{array}$ & $\begin{array}{c}p-S \\
(\%)\end{array}$ & $\begin{array}{c}p-L \\
(\%)\end{array}$ & $\begin{array}{c}p \text {-Group } \\
(\%)\end{array}$ \\
\hline Sq. ca. & $2(7)$ & $5(14)$ & $7(11)$ & $4(15)$ & $1(5)$ & $5(10)$ \\
Ad. ca. & $36(20)$ & $63(28)$ & $102(22)$ & $32(19)$ & $51(26)$ & $83(22)$
\end{tabular}

$c-S$, Clinical small (roentgenographically measured tumor diameter $2 \mathrm{~cm}$ or less); $c-L$, clinical large (roentgenographically measured tumor diameter 2.1 to $3 \mathrm{~cm}$ ); $c$-Group, c-S + c-L; $p$-S, postoperative small (tumor diameter of resected specimen $2 \mathrm{~cm}$ or less); $p-L$, postoperative large (tumor diameter of resected specimen 2.1 to $3 \mathrm{~cm}$ ); p-Group, p-S + p-L; $S q$. $c a$., squamous cell carcinoma; $A d$. $c a$., adenocarcinoma.

lower than that of group p-L. Rates were, however, variable in the postoperative group, with three positive cases in group c-L showing a change in diameter postoperatively. Two patients shifted to group $\mathrm{p}-\mathrm{S}$, and one patient shifted to the more than $3 \mathrm{~cm}$ in diameter group. Thus the positive rate of group $\mathrm{p}-\mathrm{S}$ was higher than that of group $\mathrm{p}-\mathrm{L}$.

In squamous cell carcinoma no intrapulmonary metastasis, dissemination, or malignant effusion was recognized, such that the risk rate for incomplete resection was the same as that of lymph node metastasis (Table VI). In every group the risk rate for incomplete resection in adenocarcinoma was higher than that of squamous cell carcinoma, whereas the risk rate of squamous cell carcinoma in group c-S was significantly lower than that of adenocarcinoma in group c-L $(p=0.03)$.

Postoperative survivals were calculated with the inclusion of all deaths. The 5-year survival was $79.5 \%$ in group c-S, $69.3 \%$ in group c-L, $79.2 \%$ in group $\mathrm{p}-\mathrm{S}$ and $70.4 \%$ in group p-L (Fig. 2). The survival curve of group c-S was significantly higher than that of group c-L $(p=0.02)$, and the survival curve of group $\mathrm{p}-\mathrm{S}$ was higher than that of group $\mathrm{p}-\mathrm{L}$ $(p=0.04)$. Results of multivariative analysis of the prognostic factors are shown in Table VII. The existence of risk factors is significant and tumor size is not. Excluding patients with risk factors, 5-year survival was $79.8 \%$ in group p-L $(n=170), 88.9 \%$ in group p-S ( $n=166)$, and the survival curve of group $\mathrm{p}-\mathrm{S}$ was significantly higher than that of group $\mathrm{p}-\mathrm{L}$ $(p=0.01)$.

\section{Discussion}

In 1987 the Japanese Ministry of Health and Welfare decided to include lung cancer screening with plain chest $\mathrm{x}$-ray films and sputum cytologic examination as one of the cancer screening programs provided by the Health and Medical Services Law for the Aged. With the spread of this program,
Table VII. Multivariate analysis of prognostic factors

\begin{tabular}{llrrr}
\hline Prognostic factors & Difference measured & $p$ & $H R$ & $95 \%$ CI \\
\hline Tumor size & 2 cm or less, more & 0.16 & 1.34 & $0.88-2.05$ \\
& $\quad$ than 2 cm & & & \\
Risk factors & Not exist, exist & $<0.01$ & 6.73 & $4.46-10.15$ \\
Histologic type & $\begin{array}{l}\text { Others, Ad. ca., } \\
\text { Sq. ca. }\end{array}$ & 0.06 & 1.96 & $1.21-3.18$ \\
& S. & & \\
\hline
\end{tabular}

Risk factors, Lymph node metastasis, pulmonary metastasis, pleural dissemination, or malignant effusion; $H R$, hazard ratio; $C I$, confidence interval; $A d$. ca., adenocarcinoma; $S q$. ca., squamous cell carcinoma.

identification of patients with peripheral small-sized lung cancer with a diameter of $2 \mathrm{~cm}$ or less has been increasing. However, it is not easy to diagnose these small-sized lung cancers in the preoperative period. In the reports by Cortese and McDougall $^{2}$ and Stringfield and coworkers, ${ }^{3}$ the cytologic positive rate by bronchoscopy in peripheral small-sized lung cancer was low (i.e., less than 50\%). Mori and coworkers ${ }^{4}$ reported that they detected $68 \%$ of these cases, $66 \%$ by a fiberoptic bronchoscope and $2 \%$ by needle biopsy. In our institution we were able to diagnose $87 \%$ of small-sized lung cancers, with $12 \%$ detected by percutaneous needle biopsy. Thus it is useful to conduct percutaneous needle biopsy for these small-sized lung cancers.

In all our patients with lung cancer, clinical staging was done roentgenographically, with plain chest $\mathrm{x}$-ray films and computed tomographic scanning, before the operation. The patients with tumors estimated to be cT1 N0 M0, stage I preoperatively and judged pathologically to be stage I accounted for only $78 \%$; those estimated to be peripheral small-sized cT1 N0 M0 preoperatively and judged pathologically to be stage I accounted for $82 \%$. The major cause of changes in pathologic staging was the existence of lymph node metastasis.

The focus of this study was cT1 N0 M0 peripheral non-small-cell lung cancer. Lymph node metastasis was recognized in $21 \%$ of tumors $3 \mathrm{~cm}$ or less on the basis of roentgenographic size and in $20 \%$ of tumors $3 \mathrm{~cm}$ or less measured postoperatively. Asamura and coworkers ${ }^{5}$ reported the lymph node metastasis rate to be $22 \%$ in cN0 non-small-cell lung cancers $3 \mathrm{~cm}$ or less, and Tateishi and coworkers ${ }^{6}$ reported the metastasis rate to be $30 \%$ in any $\mathrm{cN}$ non-small-lung cancers $3 \mathrm{~cm}$ or less. Therefore it was suspected that lymph node metastasis of cT1 N0 M0, peripheral, non-small-cell lung cancer was approximately $20 \%$. Comparing tumors $2 \mathrm{~cm}$ or less with those 2.1 to 3 $\mathrm{cm}$, the metastasis rate was $20 \%$ in the former and 


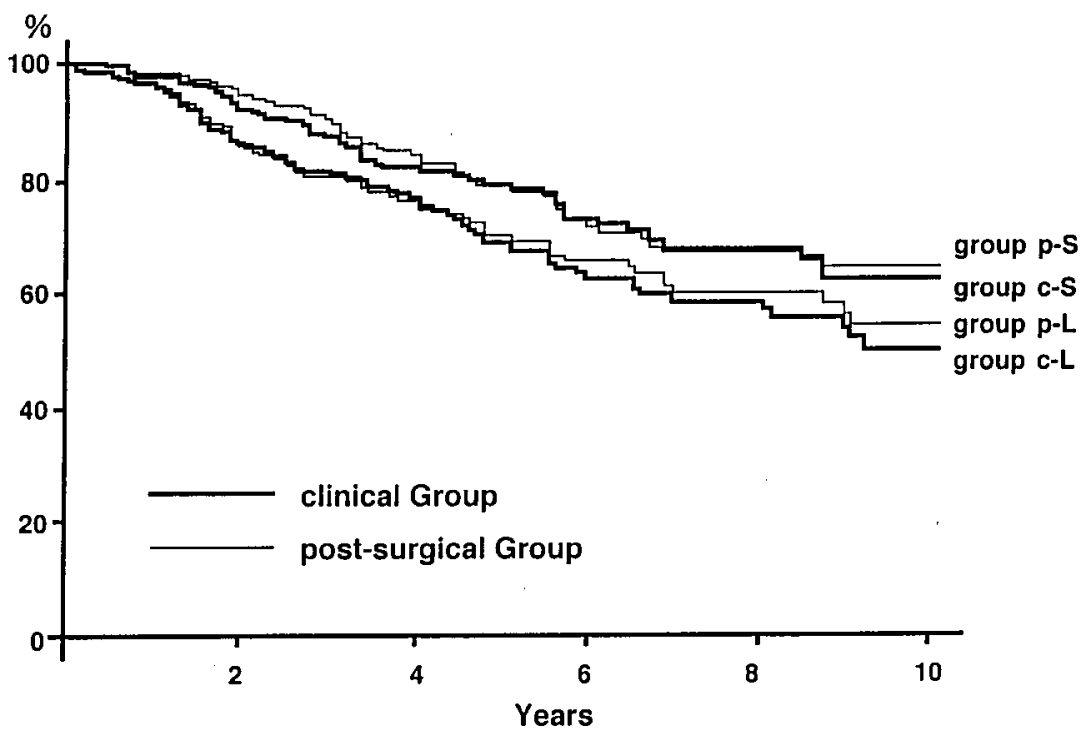

Fig. 2. Postoperative survival curve. The survival curve of group c-S was significantly higher than that of group c-L $(p=0.02)$, and the survival curve of group $\mathrm{p}-\mathrm{S}$ was higher than that of group $\mathrm{p}-\mathrm{L}(p=0.04)$.

$33 \%$ in the latter. ${ }^{5}$ Ishida and coworkers ${ }^{7}$ reported that the metastasis rate was $15 \%$ for tumors $2 \mathrm{~cm}$ or less and $38 \%$ for those in 2.1 to $3 \mathrm{~cm}$. Among our patients the metastasis rate was $17 \%$ or $18 \%$ for patients with small-sized lung cancers and $23 \%$ for those with tumors 2.1 to $3 \mathrm{~cm}$, but the difference between the two groups did not reach statistical significance.

According to histologic type, among the patients with any $\mathrm{cN} 3 \mathrm{~cm}$ or less, Asamura and coworkers ${ }^{5}$ reported the lymph node metastasis rate to be $17 \%$ in adenocarcinoma and $14 \%$ in squamous cell carcinoma. In our study, among the patients with $\mathrm{cN} 03$ $\mathrm{cm}$ or less, the metastasis rate was $22 \%$ in adenocarcinoma and $11 \%$ in squamous cell carcinoma. Among the patients with tumors $2 \mathrm{~cm}$ or less, Asamura and coworkers ${ }^{5}$ reported that the metastasis rate was $11 \%$ in adenocarcinoma and $6 \%$ in squamous cell carcinoma. The corresponding rates in our series were $17 \%$ and $15 \%$. Thus the lymph node metastasis rate of squamous cell carcinoma was lower than that of adenocarcinoma.

Intrapulmonary metastasis existed in $1 \%$ to $2 \%$, and pleural dissemination or malignant effusion was recognized in $1 \%$ to $3 \%$. In those with lymph node metastasis, pulmonary metastasis, pleural dissemination, or malignant effusion, limited resection is not appropriate. The risk rate for incomplete resection was predicted to be $18 \%$ to $19 \%$ in small-sized lung cancer and $21 \%$ to $23 \%$ with 2.1 to $3 \mathrm{~cm}$ tumors. Furthermore, according to histologic type, there was a low incidence of squamous cell carcinoma.

Comparing the survival rate of the group with tumors $2 \mathrm{~cm}$ or less in diameter with that of the group whose tumors were 2.1 to $3 \mathrm{~cm}$ in diameter, Asamura and coworkers ${ }^{5}$ reported that there was no difference between the two groups in those without lymph node metastasis. In our study, however, there were significant differences between the two groups. Recently, Motta ${ }^{8}$ reported in a review describing the current state in Europe that there was a definitive difference in postoperative survival between pT1 N0 and pT2 N0. It was therefore suspected that the postoperative survival with small-sized lung cancer was better than that associated with tumors 2.1 to 3 $\mathrm{cm}$ in diameter. There have been several reports $^{7,9,10}$ indicating that the survival of patients with small-sized lung cancer was better than that of those with 2.1 to $3 \mathrm{~cm}$ tumors. The 5-year survival of the 84 patients with 1.1 to $2 \mathrm{~cm}$ diameter tumors was $74 \%$ and was thus better than that of 129 patients with 2.1 to $3 \mathrm{~cm}$ cancers reported by Ishida and coworkers. $^{7}$ Read and coworkers ${ }^{9}$ reported that among T1 N0 cases undergoing several different operative procedures, the survival of 146 patients with tumors $2 \mathrm{~cm}$ or less was significantly better than that of 98 patients with tumors $2 \mathrm{~cm}$ or more. Warren and coworkers ${ }^{10}$ reported that the survival of patients with cancers $2 \mathrm{~cm}$ or less was better than 
those with tumors 2.1 to $3 \mathrm{~cm}$. Thus it is our view that among $\mathrm{T} 1 \mathrm{~N} 0$ cases, the survival of patients with small-sized lung cancer (i.e., a diameter of $2 \mathrm{~cm}$ or less) is better than that of other $\mathrm{T} 1 \mathrm{~N} 0$ cases.

In conclusion, in the cT1 N0 group, non-small cell lung cancer, lymph node metastasis was recognized in $21 \%$ and there was a possibility of progressive disease in $23 \%$. With small-sized lung cancers having a diameter of $2 \mathrm{~cm}$ or less, lymph node metastasis was recognized in $18 \%$, and the possibility of progressive disease in $19 \%$. According to histologic findings, squamous cell carcinoma had a lower rate of lymph node metastasis and was less often a progressive disease than adenocarcinoma.

\section{REFERENCES}

1. Mountain CF. A new international staging system for lung cancer. Chest 1986;89:225s-33s.

2. Cortese DA, McDougall JC. Biopsy and brushing of peripheral lung cancer with fluoroscopic guidance. Chest 1979;75:141-5.

3. Stringfield JT, Markowitz DJ, Bentz RR, Welch MH, Weg
JG. The effect of tumor size and location on diagnosis by fiberoptic bronchoscopy. Chest 1977;72:474-6.

4. Mori K, Yanase N, Kaneko M, Ono R, Ikeda S. Diagnosis of peripheral lung cancer in cases of tumors $2 \mathrm{~cm}$ or less in size. Chest 1989;95:304-8.

5. Asamura H, Nakayama H, Kondo H, Tsuchiya R, Shimosato Y, Naruke T. Lymph node involvement, recurrence, and prognosis in resected small, peripheral, non-small-cell lung carcinomas. J Thorac Cardiovasc Surg 1996;111:1125-34.

6. Tateishi M, Fukuyama Y, Hamatake M, Kohdono S, Mitsudomi T, Ishida T, et al. Characteristics of non-small cell lung cancer $3 \mathrm{~cm}$ or less in diameter. J Surg Oncol 1995;59:251-4.

7. Ishida T, Yano T, Maeda K, Kaneko S, Tateishi M, Sugimachi K. Strategy for lymphadenectomy in lung cancer three centimeters or less in diameter. Ann Thorac Surg 1990;50: 708-13.

8. Motta G. Recent advances in lung cancer surgery in Europe. Lung Cancer 1996;16:1-11.

9. Read RC, Yoder G, Schaeffer RC. Survival after conservative resection for T1 N0 M0 non-small cell lung cancer. Ann Thorac Surg 1990;49:391-400.

10. Warren WH, Faber LP. Segmentectomy versus lobectomy in patients with stage I pulmonary carcinoma. J Thorac Cardiovasc Surg 1994;107:1087-94.

\section{ON THE MOVE?}

Don't miss a single issue of the journal! To ensure prompt service when you change your address, please photocopy and complete the form below.

Please send your change of address notification at least six weeks before your move to ensure continued service. We regret we cannot guarantee replacement of issues missed due to late notification.

\section{JOURNAL TITLE:}

Fill in the title of the journal here.

\section{OLD ADDRESS:}

Affix the address label from a recent issue of the journal here.

\section{NEW ADDRESS:}

Clearly print your new address here.

Name

Address

City/State/ZIP
COPY AND MAIL THIS FORM TO:

Periodical Subscription Services

Mosby, Inc.

11830 Westline Industrial Dr.

St. Louis, MO 63146-3318
OR FAX TO:

314-432-1158

N/Mosby
OR PHONE:

1-800-453-4351

Outside the U.S., call

314-453-4351 